\section{The Stethoscope- A Vector of Infection?}

To the Editor:

In the course of investigating a hospital-wide outbreak of methicillin resistant Staphylococcus aureus, 67 physicians' stethoscopes were cultured. Both the bell and the diaphragm were sampled with a sterile cotton swab, and this was immediately streaked on to Columbia Nutrient Agar (BBL), a selective medium for grampositive organisms. The catalase test was used to identify Staphylococcus, and a tube coagulation test served to distinguish between $S$. aureus and $S$. epidermidis. Staphylococci were isolated from 63 stethoscopes, and all but one were coagulase negative. The single $S$. aureus isolate was methicillin sensitive. Gram-negative bacteria were not sought in our study.

Somewhat surprised by the absence of $S$. aureus from stethoscope cultures, we then pried into physicians' cleaning habits of their stethoscopes. Of 53 physicians surveyed, 34 claimed to clean their stethoscopes with $70 \%$ isopropyl alcohol pads. Two physicians also used betadine pads. The frequency of this procedure varied greatly from twice a day to once every six months, but only four physicians cleaned their stethoscopes on a daily basis. Once a month was the preferred in terval, followed by once every one or two weeks. Some physicians cleaned their stethoscopes only after examining an unkempt patient or one overtly infected. Nineteen physicians had never cleaned their stethoscopes. There was no difference in the grampositive flora of regularly cleaned stethoscopes and those never cleaned.

This pervasive colonization of stethoscopes by $S$. epidermidis was also demonstrated in a study from Amsterdam. ${ }^{\prime}$ Other reports help to place this finding in perspective. Not only is $S$. epidermidis capable of producing serious infections in hospitalized patients, ${ }^{2}$ but it may serve as a reservoir for antibiotic resistance in $S$. aureus. $^{3,4}$

The stethoscope is usually ignored as a carrier of bacteria, but $S$. aureus, Serratia and Pseudomonas were recovered from $8 \%$ of stethoscopes in one study. ${ }^{1}$ Although stethoscopes are provided in most isolation rooms, many physicians will bring in their own, in effect wearing an eleventh ungloved finger. This practice could be minimized by furnishing better quality stethoscopes in isolation rooms. Whether stethoscopes may be incriminated as fomites still remains to be seen, but an awareness of their potential to harbor pathogenic organisms should be maintained:

\section{REFERENCES}

1. Steinberg PJ, de Hoop D: De stethoscoop als drager van pathogene micro-organismen in het ziekenhuis. Ned Tijdsehr Geneeskd 1978; 122:303-305.

2. Christensen GD, Bisno AL, Parisi JT, etal: Nosocomial septicemia due to multiple antibiotic-resistant Staphylococcus epidermidis. Ann Intern Med 1982; 96:1-10.
3. Weinstein RA, Kabins $S$, Nathan $C$, et al: Gentamicin-resistant staphylococci as hospital flora: Epidemiology and resistance plasmids. J Infect Dis 1982; 145:374-382.

4. Cohen ML, Wong ES, Falkow S: Common R-plasmids in Staphylococcus aureus and Staphylococcus epidermidis during a nosocomial Staphylococcus aureus outbreak. Antimicrob Agents Chemother 1982; 21:210-215.

Julio C. Arroyo, M.D. Chief, Infecrious Diseases Kathy Harrison, R.N. Infection Control Nurse Robert Birgenheier, R.N. Infection Control Nurse. Medical and Nursing Services W.J.B. Dorn Veterans' Hospital and University of South Carolina School of Medicine

Columbia, South Carolina

\section{Disinfection Processes of Respiratory Therapy Tubing}

To the Editor:

We were very interested in Dr. Spaulding's letter to the Editor (Infect Control 1983; 4(1):8-9) regarding the article by Dr. Townsend on "An Efficacy Evaluation of Synergized Glutaraldehyde-Phenate Solution in Disinfection Respiratory Therapy Equipment During Patient Use."

We also investiga ted the disinfection processes of respiratory therapy tubing. Our study compared the efficacy of machine-assisted chemical disinfection using a Glutaraldehyde pro- 